\title{
Bioanalysis
}

\section{Best practices for metabolite quantification in drug development: updated recommendation from the European Bioanalysis Forum}

\begin{abstract}
Metabolite quantification and profiling continues to grow in importance in today's drug development. The guidance provided by the 2008 FDA Metabolites in Safety Testing Guidance and the subsequent ICH M3(R2) Guidance (2009) has led to a more streamlined process to assess metabolite exposures in preclinical and clinical studies in industry. In addition, the European Bioanalysis Forum (EBF) identified an opportunity to refine the strategies on metabolite quantification considering the experience to date with their recommendation paper on the subject dating from 2010 and integrating the recent discussions on the tiered approach to bioanalytical method validation with focus on metabolite quantification. The current manuscript summarizes the discussion and recommendations from a recent EBF Focus Workshop into an updated recommendation for metabolite quantification in drug development.
\end{abstract}

First draft submitted: 26 January 2016; Accepted for publication: 27 April 2016;

Published online: 24 May 2016

Keywords: $\mathrm{EBF} \bullet \mathrm{ICH}$ M3 (R2) • metabolite quantification • MIST • scientific validation

In 2010, the European Bioanalysis Forum (EBF) published a recommendation on best practices for metabolite quantification in relation to the principles of the tiered approach [1].

This publication focused on the ongoing discussions within the EBF on the tiered approach best practices for metabolite quantification introduced at the Crystal City III meeting [2].

With the ICH M3(R2) guidance becoming effective in 2009 [3], the team temporarily suspended the discussions on the general tiered approach and focused on providing the industry with an initial framework to meet the increasing need for quantification of metabolites in early preclinical and clinical studies as required by the Guidance. The EBF recommendation proved to be useful and was often referred to as a cornerstone in the industry discussions on metabolite quantification. At the same time, it was endorsed by the Health Authorities [4,5]. Gradually, more scientists and professional groups published strategic papers relating to the practical consequences of ICH M3(R2) [6-8] and other emerging guidelines relating to metabolite research such as drug-drug interactions (DDI) [9-11].

Subsequently, the discussion on the tiered approach continued not only within the EBF, but also within other cross-company organizations like the Global Bioanalysis Consortium (GBC) [12] and the Japan Bioanalysis Forum (JBF), as well as through publications of individual scientists [13-15]. As both industry and the heath authorities (HA) developed greater understanding and acceptance of which parts of the drug development program were within the scope of a tiered approach, new opportunities arose to extend and refine this flexibility to include bioanalytical method validation. Introducing more descriptive nomenclature highlighting the scientific validity of tiered approach practices, for example, 'scientific
Philip Timmerman ${ }^{*, 1}$, Stefan Blech'2, Stephen White ${ }^{3}$, Martha Green ${ }^{4}$, Claude Delatour ${ }^{5}$, Stuart McDougall ${ }^{\ddagger}, 6$, Geert Mannens', John Smeraglia ${ }^{5}$, Stephen Williams ${ }^{4}$ \& Graeme Young $^{3}$

'Janssen R\&D, Beerse, Belgium

${ }^{2}$ Boehringer-Ingelheim Pharma $\mathrm{GmbH}$

\& Co. KG, Biberach, Germany

${ }^{3}$ GlaxoSmithKline Research \&

Development, Ware, UK

${ }^{4}$ Charles River, Edinburgh, UK

${ }^{5}$ UCB BioPharma, Braine-L'Alleud, Belgium

${ }^{6}$ Covance, Alnwick, UK (at time of authoring)

*Author for correspondence:

Tel.: +32 14603581

ptimmerm@its.jnj.com

${ }^{\ddagger}$ Currently at Arcinova, Alnwick, UK 
validation', helped the bioanalytical community and their stakeholders understand the many benefits of adopting such procedures. As a consequence, by identifying metabolite quantification as an area within the scope of scientific validation [16] discussions to understand what scientific validation means within this context and the best way to apply it within the tiered approach form the basis of the current proposed recommendation.

Because of the increased focus on metabolites in safety testing (MIST) [2], the industry rapidly gained expertise on how metabolite profiling and quantification was impacting their projects, both in relation to safety and efficacy decisions taken from the data as well as in relation to the resources required to generate metabolite concentration data in the bioanalytical (BA) or drug metabolism \& pharmacokinetics (DMPK) laboratory. As a result of the latter, while the bioanalytical community created awareness of the possibility of quantifying multiple metabolites in multiple studies, it also challenged an industry attitude to quantify metabolites in too many studies just 'because we can' (i.e., the methodology allowed it). In a collaborative effort, they looked at better ways to generate the plasma (or by extension serum, blood, tissue, urine,...) concentration data needed to make project decisions: combining better ways to interpret the different Guidances surrounding the scientific questions related to MIST and to provide exposure data in different phases of clinical development. The goal was to rationalize which metabolites should be quantified, at which phase of development and with what level of scientific and regulatory rigor as a project moves forward in the development pipeline. Essential in this discussion was a correct understanding of the regulatory requirements from an ICH M3 (R2) and DDI perspective, combined with applying the right level of bioanalytical validation in line with the stage of development (i.e., scientific vs regulatory validation) [5,16-18].

One of the challenges was how to implement a new Guideline in an industry where decisions made following this Guideline will only impact a project 5-10 years later. In the absence of rapid feedback from the HA, the resulting behavior is often to do more than is needed to be sure any risk is minimized, irrespective of the added scientific value. However, this is a sustainable approach only if applied in late stage development when attrition has decimated the number of projects to which the work needs to be applied. But, for ICH M3 (R2) the challenge lies in the disjunction between the timing when the data are needed from a regulatory perspective versus the best timing in a project based on the scientific need and availability of the study samples to generate those data. Indeed, ICH M3 (R2) only requires the metabolite coverage (expression and exposure) information to be available at the start of clinical Phase III (from ICH M3 (2): "Nonclinical characterization of a human metabolite(s) is only warranted when that metabolite (s) is observed at exposures greater than $10 \%$ of total drug-related exposure and at significantly greater levels in humans than the maximum exposure seen in the toxicity studies. Such studies should be conducted to support Phase III clinical trials.") [3]. But the study samples needed to make those decisions related to metabolite coverage across species are typically available in an earlier stage of development, for example, at the firstinto-man (FiM) and multiple ascending dose (MAD) studies when samples from 1 or 3 months GLP toxicology studies are available. As a consequence, the industry is tempted to de-risk their projects with respect to metabolite coverage at an earlier stage of development than required for ICH M3 (R2) and is therefore investing a lot of resources in projects that will never reach the clinical Phase III stage.

With this awareness, we deemed it necessary to revisit our 2010 recommendation paper [1]. In the initial recommendation, we identified a significant number of early development studies in scope to be analyzed using regulatory validation where, based on current thinking on the tiered approach/scientific validation, alternative leaner methodologies will be equally valid to make the right project decision. Supported and stimulated by the feedback from experts in the drug metabolism arena, the EBF issued a survey on the topic and organized a Focus Workshop in September 2015 in Brussels to discuss and propose a refined recommendation for metabolite quantification. At the Workshop we also took the opportunity to discuss the current practice of metabolite profiling. The proposed recommendation largely applies to small molecule new chemical entities (NCEs), but is appropriate for other drugs if the tools are available to characterize and quantify their metabolites.

\section{Feedback from the survey}

The workshop was preceded by a survey in the EBF community on the topic.

In this survey, we probed the EBF community on their day-to day practices around metabolite profiling and quantification. A total of 29 companies responded. The majority of the responses related to NCEs (26 companies) and peptides ( 9 companies). The questions related to:

- Availability of a company strategy for metabolite profiling and quantification;

- The Guidance is followed; 
- The laboratory in which the analysis is performed (internally, outsourced or DMPK or BA laboratory);

- The technology platform is used;

- The samples (species) and matrices used for profiling and/or quantification;

- The regulatory rigor used for metabolite quantification;

- The timing of the work;

- Reporting.

A summary of the survey data can be found on the Focus Workshop conference website [19].

This allowed us to re-establish our community's best current practice and forward thinking on the subject.

\section{Focus workshop}

The Focus Workshop was attended by approximately 80 scientists from over 50 different companies. Both the survey feedback and the good attendance of metabolite profiling (drug metabolism scientists) and metabolite quantification experts (bioanalytical scientists) at the workshop allowed us to consider our current updated recommendation to be representative.

The first half of the workshop was built around feedback on the current status of metabolite profiling and quantification practices. Both the EBF as well as four individual (global) pharma companies presented their current strategy on the subject, followed by a CRO perspective. The second half of the meeting comprised two parallel breakout sessions. The first one discussed the updated EBF recommendation proposal on metabolite quantification; the second one discussed potential ways to strategize metabolite profiling.

\section{Workshop on metabolite quantification}

The updated EBF recommendation for metabolite quantification supported by the workshop discussions is visualized in Figure 1.

\section{Discussion}

Referring to the initial recommendation of 2010, we removed the difference in strategy for metabolites with known activity/toxicity versus unknown activity/toxicity. In general, our current thinking is that there should be no difference in strategy toward documenting relative exposures in experimental animals versus human based on activity/toxicity prior to evaluating a drug against the ICH M3(R2) Guideline. Indeed, prior to understanding the coverage in human versus experimental animals or the contribution of metabolites to the pharmacological activity, this difference seems to make little sense. In unique cases where the metabolite is known to be equipotent or of similar activity to the dosed drug, it is obvious that quantification of that metabolite should follow the same strategy as that of the dosed drug. However, and as mentioned at the workshop: 'the exception is the exception' and should not be setting the rule. Removing this difference allows a simplified decision tree, which can be more easily connected to the aforementioned discussions on alternative approaches to bioanalytical method validation.

\section{Metabolite quantification in the drug discovery} phase

In the discovery phase, we recommend the use of screening methods for metabolite quantification [1]. Also, and connected to the fact that more requests for metabolite quantification are entering the bioanalytical laboratory in this early phase, we suggest to scrutinize these requests to balance the workload of metabolite quantification versus the added value of these additional data when making project decisions. This does not imply we advise against metabolite quantification in general in the discovery phase, but it makes sense to challenge the request to the extent that the added workload is understood by the project representatives.

\section{Metabolite quantification in pre-Phase I \\ \& early clinical phase}

This is an area of major change to our recommendation. Our update aligns both with the discussions at the workshop and the two major surveys held in 2015, and another related to the areas of application of scientific validation in support of the recent EBF recommendation paper [16]; this latter survey was re-issued after refinement in preparation of the global discussion on the subject at the 2015 AAPS APQ Open Forum. In both surveys, we probed the EBF community on their view as to whether metabolite quantification in early development would require regulatory or scientific validation. More than $80 \%$ of the responders agreed that scientific validation is a better approach, both in the EBF community as well as in the AAPS and JBF community.

In the current updated recommendation, we propose to use either screening methods (which includes relative comparison of metabolite abundance - peak area ratios of metabolites/dosed drug across species) or scientifically validated method (see Box 1) for bioanalysis in support of assessing the exposure of metabolites in man relative to experimental animals.

Several strategies can be applied to the timing of the experiments based on the availability of samples. Three 


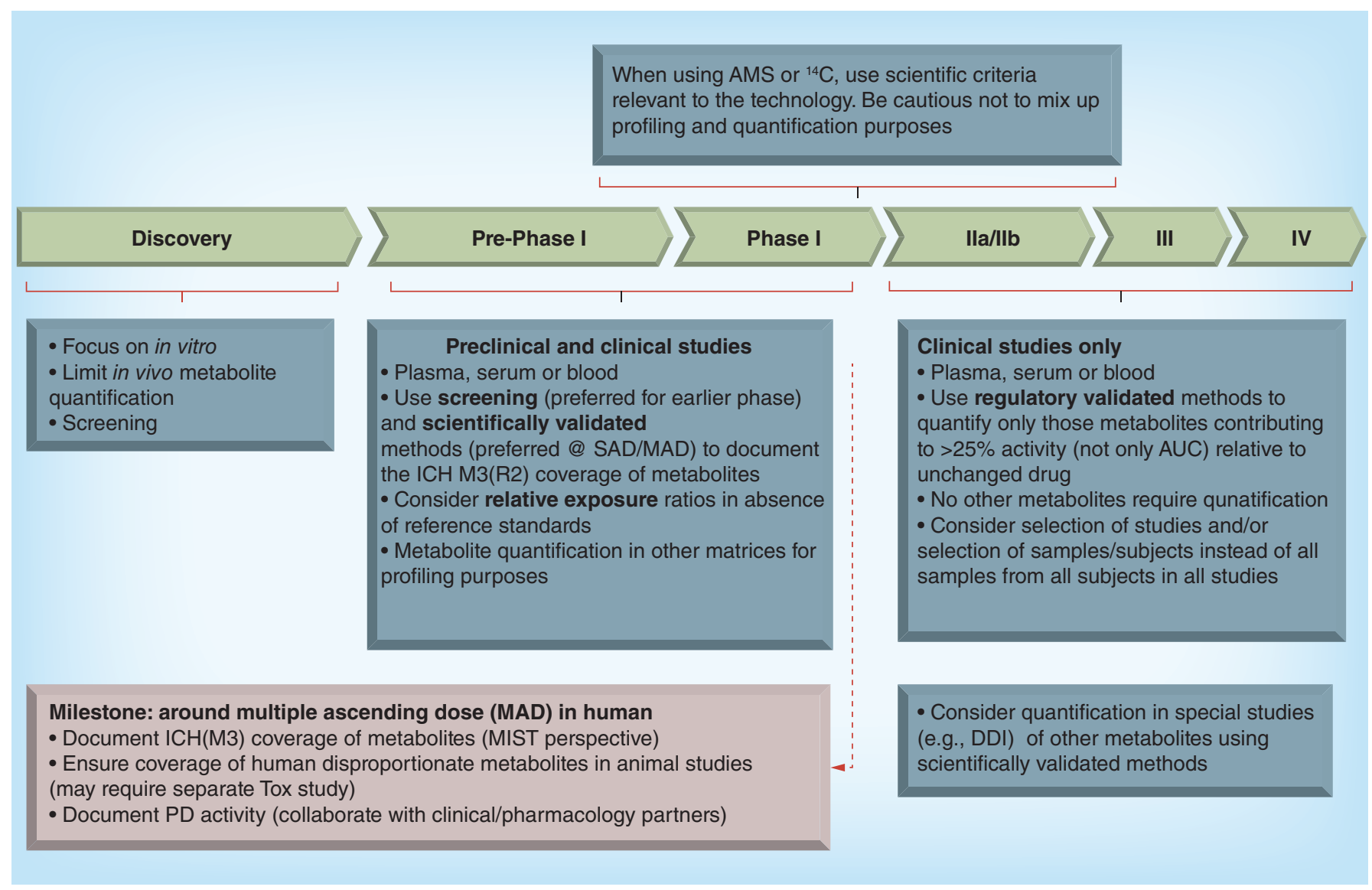

Figure 1. Updated EBF recommendation for metabolite quantification.

major approaches are common in the industry today, the latter being the most common approach:

- Samples from earlier toxicokinetic (TK) studies are stored, with the analysis to be included in this single assay campaign at the time human samples become available.

- Samples from more recent TK studies are included in a single assay campaign when samples from the multiple ascending dose study are available.

- Metabolite concentration data in the different species and human are generated at the time these samples become available.

In any strategy that is followed, the bioanalytical scientist needs to ensure that the matrix concentrations are supported with documented and reconstructable assay performance data on accuracy, precision and stability in line with the purpose of the decision to be made. We suggest using the scientific validation criteria for those metabolites for which a reference standard is available. However, strictly speaking, at this stage, there is no need to have access to a reference standard as long as a relative assessment comparing metabolite/dosed drug peak areas across species can be made with similar scientific rigor and considering relevant matrix stability data.

Although ICH M3(R2) only requires a metabolite strategy at the start of Phase III, it is likely to be more practical for the industry to perform this metabolite coverage assessment at the time the samples from multiple dosing studies are available. Considering a standard study package in the development process of a drug, we usually have access to samples from 1-month or 3-month multiple dose studies in the main toxicology species at the time of the MAD study. So, from a practical perspective and provided stability data can be generated for the metabolite (ratio), it makes sense to perform this experiment around that time frame in a single campaign.

The result of the analysis is either 'coverage' or 'no coverage'.

- The desirable outcome is that there is metabolite coverage in the preclinical species. From here onward, the strategy can become simple: stop all future analysis of metabolites in preclinical species. For man, we still need to factor in the potential contribution to the pharmacological activity and 


\section{Box.1 Definitions for regulatory and scientific validation.}

- Regulatory validation: Assay validations to provide scientifically accurate, reproducible and reconstructable concentration data to allow valid decision making for the intended purpose of the study and comply with regulated BA standards as specified by health authority (HA) guidance documents

- Scientific validation: Assay validations to provide scientifically accurate, reproducible and reconstructable concentration data to allow valid decision making for the intended purpose of the study and can withstand independent review - including scientific review from regulators if so required - although not applying all elements specified by HA guidance documents

the DDI potential. This is discussed in the next paragraph, but is irrelevant for preclinical studies.

- For metabolites where there is no coverage in man, we suggest updating the development plan and include studies/experiments to ensure coverage in preclinical species is documented (e.g., separate dosing of the metabolite or higher doses of the investigational drug to reach higher exposure levels of the metabolites, if ethically acceptable). In some cases (e.g., dosing a metabolite in a GLP tox study) under the current paradigm of bioanalytical method validation, a regulatory validated method may be required to quantify these metabolite concentrations. Moving forward, we are encouraging the industry and regulators to consider accepting scientific validation in early development GLP studies, with the view that the proposed scientific rigor, a priori set criteria and level of documentation are aligned with scientific and GLP requirements. However, although discussed at the workshop, this is not the subject of the current recommendation paper.

In addition to metabolite coverage from an $\mathrm{ICH}$ M3(R2) perspective, it is important in the process to include additional characteristics of the metabolite beyond the exposure (i.e., AUC at steady state) to fully understand the contribution of the metabolite to the overall activity. Often assessments are made on AUC only, but it is recommended to include those parameters which describe the contribution to the activity as well. Although this is an area in need of intensified discussion, a proposed starting point would be to include at least the pharmacological activity (if available) or plasma protein binding of the metabolite(s) and the dosed drug to correct the AUC for activity. It is our recommendation that, in addition to not requiring further quantification of metabolites when we have either coverage in preclinical species or the exposure of a metabolite in human plasma is less than $10 \%$ of total drug exposure, also to preclude the requirement for further quantification of metabolites when the relative contribution to the activity in man is less than $25 \%$ compared with the dosed drug, (based on steady-state AUC values corrected for pharmacological activity).
In summary, and in contrast to our initial recommendation, the EBF does not see a requirement to use regulatory validated methods for metabolites until after the MAD study, for either preclinical or clinical studies. All metabolite quantification and decisions on coverage of metabolites in relation to $\mathrm{ICH}$ M3(R2) can be documented using screening and scientific validation approaches. Also, if metabolite exposure in man is covered in preclinical species, we recommend stopping all further analysis of metabolites in preclinical studies.

\section{Continued metabolite quantification after the multiple ascending dose}

This is the second area of change in comparison to our initial recommendation, and is likely one with significant impact on the bioanalytical laboratory. The current regulatory requirements for metabolite coverages in man versus experimental animals, stating: "Nonclinical characterization of a human metabolite(s) is only warranted when that metabolite(s) is observed at exposures greater than $10 \%$ of total drug-related exposure and at significantly greater levels in humans than the maximum exposure seen in the toxicity studies." [3] equally provides guidance on how to continue the metabolite quantification in later stages of development.

First, and as recommended above, if the metabolite coverage is documented and complies with $\mathrm{ICH}$ M3(R2), there is no need for continued quantification of metabolites in preclinical species. Hence, and since our recommendation is to perform this assessment at the MAD milestone, there is no need to continue the quantification of metabolites in preclinical species after this milestone. At the same time, there is also little value in excessive quantification of metabolites prior to this assessment, since the assessment qualifies the need for metabolite quantification.

On a case-by-case basis there may be a need to include additional toxicology species at later stages in development. In those cases we would recommend analyzing metabolites, if needed, using the screening (including relative peak area ratios of metabolite/dosed drug) or scientifically validated approach as detailed earlier.

With respect to continued quantification in human samples, a similar rationale is proposed. And although 
it is very tempting for project teams to continue to request metabolite concentrations in later clinical studies, it is our recommendation to only generate metabolite concentrations for those metabolites that contribute to more than $25 \%$ of the activity in relation to the dosed drug (based on AUC corrected for the pharmacological activity) in the continuation of the development of the drug. In essence we do recommend using regulatory validated methods for all metabolites complying with these characteristics. We would however consider not necessarily continuing quantification of the metabolites in all studies but only in a selection of studies and/or selection of samples/subjects.

There are two areas of concern:

- There are programs in which the drug is extensively metabolized (e.g., prodrugs or hormones are good examples). In those cases, the above recommendation can lead to an explosion of metabolites qualifying for continued quantification and it may be warranted to agree on an alternative strategy. Agreeing beforehand on the bioanalytical strategy to be followed with the HA is advised. Both the sponsor and the HA should be open to a strategy which considers understanding the PK/PD relationship while appropriately limiting the resource investments required to generate these data. Strategizing the BA work around a major pharmacological metabolite can be a fair starting point.

- Special population studies (e.g., elderly, hepatic/renal impaired and children) and DDI studies where, depending on differences in clearance and/or metabolism, metabolite exposure could increase above the thresholds mentioned in the ICH Guidance. If known metabolites of the investigational drug would need to be measured in those studies, we would recommend using scientific validation and focus on those metabolites which in an earlier assessment were on the borderline of being covered and for which there may be a concern. Again we would recommend to consider only quantifying metabolites in a selection of samples/subjects. For the analysis of metabolites from the industry standard DDI probes, we recommend regulatory validated methods.

In summary, after the MAD study, identified as the milestone to document metabolite coverage in relation to ICH M3(R2), we recommend to only quantify those metabolites that contribute more than $25 \%$ to the pharmacological activity compared with the dosed drug. Quantification of those metabolites should be performed using a regulatory validated method. We recommend evaluating whether the metabolite(s) need(s) to be included in every study from that stage onward, and at which stage enough data are gathered. Any metabolites that require quantification in special clinical or preclinical studies would only require a scientific validation. For an extensively metabolized drug, other strategies may apply.

\section{Conclusion for metabolite quantification}

In this updated recommendation on metabolite quantification in drug development, we recommend limiting quantitation of metabolites in all stages of development to those that are needed to understand their contribution to the activity or toxicity of the dosed drug. Also, we recommend using the principles of tiered approach for bioanalytical method validation.

For the different stages of development, this means:

- Drug discovery: minimize the analysis of metabolites in in vivo samples. If analysis is required, use screening methods, including relative peak area ratios of metabolites/dosed drug.

- Early development (pre-Phase 1 until MAD): assess metabolite exposure in man and coverage in preclinical species at the timing of the MAD compared with metabolite exposure in multiple dose preclinical studies. Use screening methods, including relative peak area ratios of metabolites/dosed drug, or scientific validation.

- After MAD: no need to quantify any metabolites in preclinical species unless required by ICH M3(R2). In clinical studies, quantify only those metabolites contributing to $>25 \%$ activity (based on AUC corrected for activity) relative to dosed drug using regulatory validated methods and consider selection of studies and/or selection of samples.

- Treat exceptions as exceptions: any metabolites requiring quantification in special clinical or preclinical studies would only require a scientific validation. For extensively metabolized drug, other strategies may apply. In special cases, agree on a bioanalytical strategy, preferably with the HA based on scientific needs and sustainable use of resources.

A schematic overview is presented in Figure 1.

\section{Workshop on metabolite profiling}

The discussions related to metabolite profiling tried to unite the audience around the strategy used for metabolite profiling: "Who does What, When, Why and How?" Then build on the input from the aforementioned survey [19].

From the survey, Figures 2 \& 3 were developed. The figures show at what stage the DMPK laboratory gets 


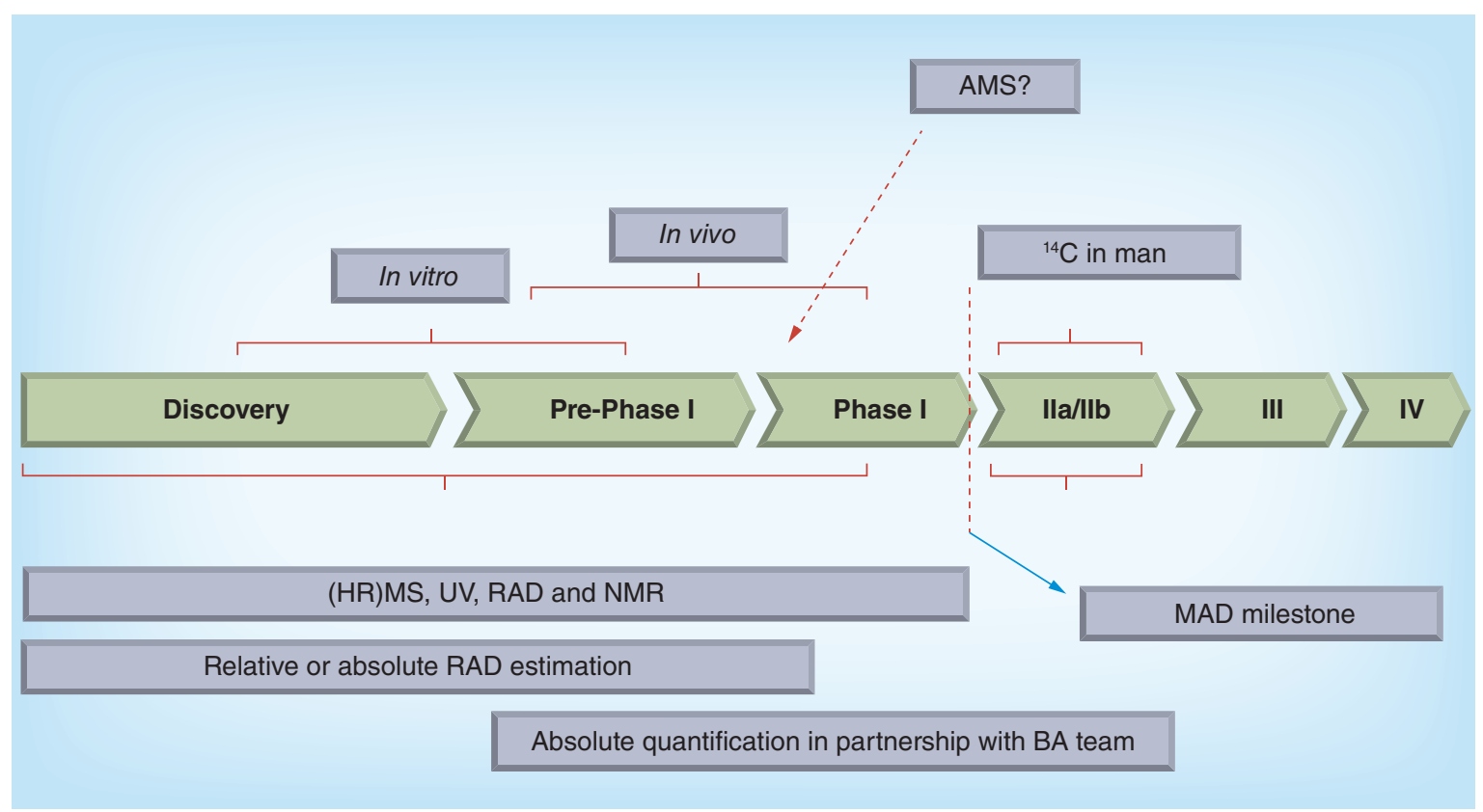

Figure 2. Metabolite profiling strategies in drug development: tools involved.

involved, which analytical tools are used for profiling and which types of in vivo and in vitro studies are performed for small molecule (NCE) metabolite profiling. This information, together with the presentations from four Pharma companies and a CRO were the starting point of the workshop discussions on metabolite profiling. In contrast to metabolite quantification, where the audience could build on an existing EBF Recommendation on top of many years of intense discussion in the industry, metabolite profiling was identified as an area where it will be difficult to provide a similar recommendation. Every molecule is different and requires scientific freedom in regard to documentation and development of understanding of its metabolic fate. Hence, our expert audience focused on sharing ideas, experience and strategies on how the various technologies in different stages of development add value.

\section{Highlights from the discussions}

In addition to sharing individual company strategies as a source of inspiration for metabolite profiling, the workshop discussion covered four main areas:

- General considerations

- Regulatory aspects

- Quality aspects

- Scientific aspects - with focus on the complexity of metabolite stability during sampling storage and sample handling in multiple matrices involved in metabolite profiling.
The delegates rapidly identified that because of the absence of regulatory guidance related to metabolite profiling and the split between the more traditional front loaded approach (i.e., a relatively large package of work conducted preclinically often using radiolabeled drug) versus a 'human first' based approach (with more limited support work conducted pre-FTIH and radiolabel not used until much later in development) it is difficult to provide guidance on this subject. The governance in a company should drive the strategy. Hence, we limit our feedback on the metabolite profiling workshop to state that it is essential to allow scientific freedom and continue to benefit from the rapidly evolving technological innovations.

All the presentations from the meeting and bulleted highlights from the workshop can be found on the EBF Focus Workshop website [20]. They illustrate how companies strategies can differ in their approaches yet are able to generate the required information in time to make adequate project decisions. All roads apparently lead to Rome, although it was not built in a day. The latter is certainly also true for elucidation of the metabolism of many compounds in industry.

In addition, the discussions at the workshop provided an opportunity to connect DMPK experts with the ongoing discussions in the bioanalytical arena on scientific validation. In this respect, it is important to highlight scientists in both functional teams are involved in quantification of metabolites, yet using different quality standards or processes. Often, the analytical acceptance criteria in the DMPK labora- 


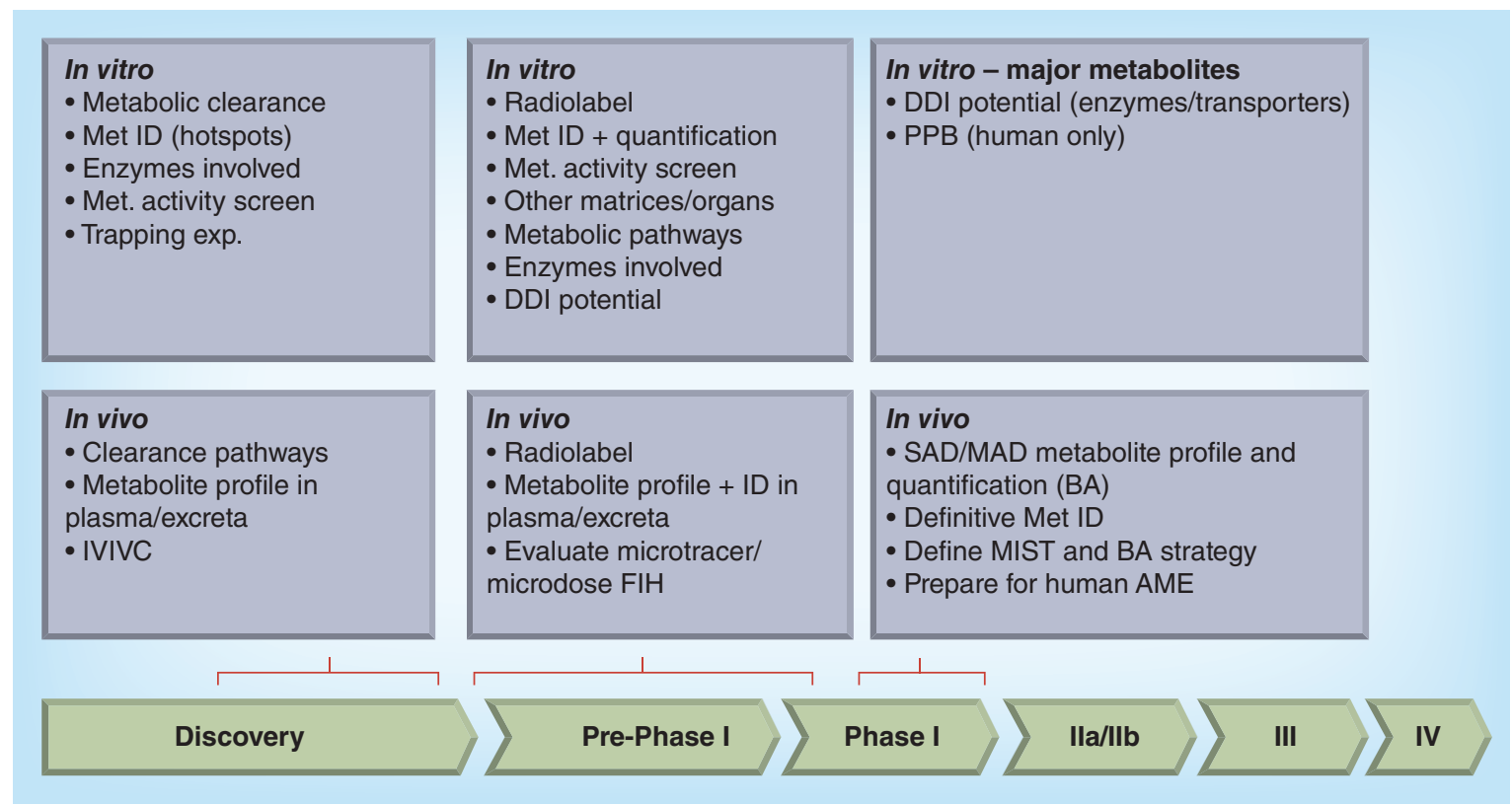

Figure 3. Metabolite profiling strategies in drug development: studies involved.

tory are not governed by internationally agreed quality standards as compared with regular bioanalysis. Nevertheless, these metabolite profiling activities do follow scientifically valid criteria in line with the specific technology used (and often described in company SOPs or procedural documents), be it ultraviolet (UV) detection, accelerator mass spectrometry (AMS), radioactive detection (RA), NMR (nuclear magnetic resonance) or other tools. This manuscript is not intended as a plea for more regulations in the area of metabolite quantification embedded in metabolite profiling activities. We do want to highlight that a close(r) partnership between the metabolite profiling scientists in the DMPK laboratory and the bioanalytical laboratory is essential to understand acceptance criteria and to avoid potentially incorrect interpretation of the accuracy of concentration data originating from RAD, UV or MS which can, if so required, be annotated appropriately.

\section{Conclusion}

Over the last 5 years, the industry has gained a lot of experience with the practical aspects, including the use of the data in drug R\&D, of metabolite profiling and quantification. This, together with the intensified discussion and added value of the principle of the tiered approach and scientific validation in bioanalysis has stimulated the EBF to take a fresh look at their recommendation on metabolite quantification from 2010. As a result of new targeted surveys and peer discussion at a Focus Workshop in September 2015, the EBF is now refining their 2010 recommendation. In the updated recommendation, we are putting more emphasis on the value of scientific validation approaches as an alternative to regulatory validation for metabolite quantification in the earlier stages of development. At the same time, we recommend the industry reflects on limiting quantitation of metabolites once the contribution of metabolites to the toxicology or pharmacology is understood.

For metabolite profiling, the discussions from the workshop highlighted the importance of scientific freedom, use of state-of-the-art technology and increased partnership between drug metabolism experts and bioanalytical scientists as a good recipe for a result-driven strategy.

\section{Acknowledgements}

The authors want to acknowledge the EBF member companies that have contributed to the surveys, workshops and internal discussions, which form the backbone of the current recommendation.

\section{Disclaimer}

The views and conclusion presented in this paper are those of the European Bioanalysis Forum and do not necessarily reflect the representative affiliation or company's position on the subject.

\section{Financial \& competing interests disclosure}

The authors have no relevant affiliations or financial involvement with any organization or entity with a financial interest in or financial conflict with the subject matter or materials discussed in the manuscript. This includes employment, consultancies, honoraria, stock ownership or options, expert testimony, grants or patents received or pending, or royalties.

No writing assistance was utilized in the production of this manuscript. 


\section{References}

1 Timmerman P, Kall M, Gordon B et al. Best practices in a tiered approach to metabolite quantification: views and recommendations of the European Bioanalysis Forum. Bioanalysis 2(7), 1185-1194 (2010).

2 Viswanathan CT, Bansal S Booth B et al. Workshop/ Conference Report - Quantitative bioanalytical methods validation and implementation: best practices for chromatographic and ligand binding assays. AAPS J. 9(1), E30-E42 (2007).

3 European Medicines Agency. CPMP/ICH/286/95- ICH Topic M 3 (R2) non-clinical safety studies for the conduct of human clinical trials and marketing authorization for pharmaceuticals. London, UK (2009).

4 Booth B. When do you need a validated assay? Bioanalysis 3(24), 2729-2730 (2011).

5 Ministry of Health, Labor and Welfare. Guideline on bioanalytical method validation in pharmaceutical development. Tokyo, Japan (2013) www.nihs.go.jp/drug/BMV/250913_BMV-GL_E.pdf

6 Leclercq L1, Cuyckens F, Mannens GS, de Vries R, Timmerman P, Evans DC. Which human metabolites have we MIST? Retrospective analysis, practical aspects, and perspectives for metabolite identification and quantification in pharmaceutical development. Chem. Res. Toxicol. 22(2), 280-293 (2009).

7 Ma S, Chowdhury S. Analytical strategies for assessment of human metabolites in preclinical safety testing. Anal. Chem. 83, 5028-5036 (2011).

8 Yu H, Bischoff D and Tweedie D. Challenges and solutions to metabolites in safety testing: impact of the International Conference on Harmonization M3(R2) guidance. Expert Opin. Drug Metab. Toxicol. 6(12), 1539-1549 (2010).

9 European Medicines Agency. Guideline on Investigation of Drug Interactions. London, UK (2012). www.ema.europa.eu

10 Ministry of Health, Labor and Welfare. Guideline on Drug Interactions for Drug Development and Appropriate Provision of Drug Information (final draft.) Tokyo, Japan (2014).
11 US FDA. Guidance for Industry: Drug Interaction Studies - Study Design, Data Analysis, Implications for Dosing, and Labeling Recommendations. www.fda.gov

12 Lowes S, Hucker R, Jemal M et al. Tiered approaches to chromatographic bioanalytical method performance evaluation: recommendation for best practices and harmonization from the global bioanalysis consortium harmonization team. AAPS J. 17(1) 17-23 (2015).

13 Timmerman Philip. Mini focus issue: tiered approach to bioanalysis. Bioanalysis 6(5), 585-682 (2014).

14 Timmerman P. Tiered approach revisited: introducing stage-appropriate or assay-appropriate scientific validation. Bioanalysis 6(5), 599-604 (2014).

15 Timmerman P, Lowes S, McDougall S et al. Scientific or regulated validation: a tiered approach? Meeting report from a joint EBF/DVDMDG workshop. Bioanalysis 7(14), 1703-1710 (2015).

16 Timmerman P, White S, McDougall S et al. Tiered approach into practice: scientific validation for chromatography-based assays in early development - a recommendation from the European Bioanalysis Forum. Bioanalysis 7(18), 2387-2398 (2015).

17 US. Department of Health and Human Services Food and Drug Administration Center for Drug Evaluation and Research (CDER) Center for Veterinary Medicine (CVM). Guidance for Industry Bioanalytical Method Validation (2001). www.fda.gov/downloads/Drugs/Guidance/ucm070107.pdf

18 European Medicines Agency. Guideline on bioanalytical method validation EMEA/CHMP/EWP/192217/2009. www.ema.europa.eu

19 Feedback from the survey - Stuart Mc Dougall on behalf of the EBF. www.focus.europeanbioanalysisforum.eu

20 EBF - Focus Workshop - Bringing Assay Validation and Analysis of Biomarkers into Practice. http://focus.europeanbioanalysisforum.eu/slides/ 